\title{
A Neuro-Inspired Computational Model for a Visually Guided Robotic Lamprey Using Frame and Event Based Cameras
}

\author{
Ibrahim Youssef $^{1}$, Mehmet Mutlu ${ }^{1,2}$, Behzad Bayat ${ }^{1}$, Alessandro Crespi $^{1}$, Simon Hauser ${ }^{1}$, Jörg Conradt $^{3}$, \\ Alexandre Bernardino ${ }^{2}$, and Auke Ijspeert ${ }^{1}$
}

\begin{abstract}
The computational load associated with computer vision is often prohibitive, and limits the capacity for on-board image analysis in compact mobile robots. Replicating the kind of feature detection and neural processing that animals excel at remains a challenge in most biomimetic aquatic robots. Event-driven sensors use a biologically inspired sensing strategy to eliminate the need for complete frame capture. Systems employing event-driven cameras enjoy reduced latencies, power consumption, bandwidth, and benefit from a large dynamic range. However, to the best of our knowledge, no work has been done to evaluate the performance of these devices in underwater robotics. This work proposes a robotic lamprey design capable of supporting computer vision, and uses this system to validate a computational neuron model for driving anguilliform swimming. The robot is equipped with two different types of cameras: framebased and event-based cameras. These were used to stimulate the neural network, yielding goal-oriented swimming. Finally, a study is conducted comparing the performance of the computational model when driven by the two different types of camera. It was observed that event-based cameras improved the accuracy of swimming trajectories and led to significant improvements in the rate at which visual inputs were processed by the network.
\end{abstract}

Index Terms-Biologically-Inspired Robots, Neurorobotics, Visual-Based Navigation, Marine Robotics, Event-Based Vision

\section{INTRODUCTION}

$\mathbf{R}$ OBOTICS and biology benefit from a symbiotic relationship such that breakthroughs in one domain often lead to progress in the other [1]. The field of biology is a well

Manuscript received: September, 9, 2019; Revised December, 16, 2019; Accepted January, 17, 2020.

This paper was recommended for publication by Editor Xinyu Liu upon evaluation of the Associate Editor and Reviewers' comments. This work was supported by the Fundação para a Ciência e Tecnologia (FCT) agency of Portugal under the contract number PD/BD/105781/2014, the Swiss National Science Foundation (Project 153299), the European Commission project ORIENT (ERC/2016/693400), and has received funding from the European Union's Horizon 2020 Framework Programme for Research and Innovation under the Specific Grant Agreement No. 785907 (Human Brain Project SGA2). This work was also made possible by NCCR Robotics, the IST-EPFL program, and the Nano-Tera initiative.

${ }^{1}$ Ibrahim Youssef, Mehmet Mutlu, Behzad Bayat, Alessandro Crespi, Simon Hauser, and Auke Ijspeert are with the Biorobotics Laboratory, School of Engineering and Institute of Bioengineering, École Polytechnique Fédérale de Lausanne, Switzerland ibrahim.youssef@alumni.epfl.ch

${ }^{2}$ Mehmet Mutlu and Alexandre Bernardino are with the Electrical and Computer Engineering, Faculty of Engineering, Instituto Superior Téchnico, Lisbon, Portugal alexeisr.tecnico.ulisboa.pt

${ }^{3} \mathrm{Jörg}$ Conradt is with the School of Electrical Engineering and Computer Science, KTH Royal Institute of Technology, Stockholm, Sweden jconradt@kth.se

Digital Object Identifier (DOI): see top of this page. established bastion of inspiration for robotic development, and has had an impact on sub-domains ranging from perception and artificial intelligence to mechanical design. At the same time, biologists and neuroscientists have begun employing robotic systems to validate hypotheses and computational models in their fields [2][3]. The lamprey is one such organism at the center of this exchange, and has been the subject of extensive modelling and experimentation. Due to its simple locomotion strategy, well studied physiology, and relatively primitive nervous system, this organism remains fertile ground for similar studies in vision-guided locomotion.

This work investigated a solution to the control problem facing visually guided swimming robots. Previous research has explored the application of traditional feedback control strategies for guiding aquatic robots using visual input [4][5]. Simpler control strategies have also been used to implement goal-directed swimming in robotic lamprey [6]. More recently, a vision guided fish robot developed by Yu et al. made use of a simple body wave model to produce fish-like swimming patterns [7]. These strategies have all been used to track singular objects in the robots' fields of view. Little work has been done on robot controllers capable of resolving conflicts between competing stimuli for such aquatic robots. It would be difficult to extend existing control laws to encode and manage more complex behaviors without introducing significant complexity. The study of central pattern generators (CPGs) has gone a long way towards simplifying the problem of coordinating gaits in robotic systems [8][9]. Still, effective strategies must be developed that are capable of bridging the gap between complex visual input and CPG drive signals.

The large computational load associated with high-framerate image processing remains one of the major limitations to real-time vision controlled robots. Event-based cameras offer a potential solution to overcome many of these limitations. These event driven cameras operate in a way that resembles the function of retinal photoreceptors. Rather than producing frames at fixed intervals, this sensory strategy reports events in response to light log-intensity changes at individual pixels [10]. What is then communicated by the sensor is not a complete image, but a stream of events specifying which pixels have registered positive or negative changes in light intensity. This approach allows visual information to be transmitted at a significantly lower bandwidth since redundant information is withheld. At the same time, these systems offer a high dynamic range, making them attractive to implement high 
speed computer vision algorithms on-board robots. A growing body of research has shown how event driven sensors may be used to improve aggressive maneuvering with Unmanned Aerial Vehicles (UAVs) [11], but no work has been done to study their potential value to underwater robotics.

The two objectives of this study were to validate a biologically inspired visuomotor controller, and pioneer the use of event-driven cameras in an underwater robot. This work validated and extended the biologically inspired visuomotor control architecture proposed by Sarvestani et al. [12]. In order to evaluate the control strategy, it was reproduced and used as the main high-level controller for the Envirobot platform. The Envirobot is an anguilliform swimming robot developed by Bayat et al. [13] for the monitoring of environmental conditions in lakes. Here, multiple behaviors were implemented such that the robot was capable of navigating through an environment containing various attractive and repulsive visual cues. Finally, the frame-based cameras used to stimulate the visuomotor controller were replaced with a pair of eventbased cameras, and performance was compared in a target approach task for the two modalities. The challenge with these sensors, particularly in the unexplored domain of underwater vision, lies in the development of algorithms capable of effectively processing the visual data. Neural-network-based control strategies, such as that presented in this work, are expected to be particularly well-suited to exploiting the output properties of such event driven cameras. This marked the first instance of dynamic vision sensors being used in an aquatic environment.

\section{METHODS}

\section{A. Envirobot Design}

The Envirobot platform was upgraded to include computer vision capabilities. A NanoPi NEO Plus2 was used to capture images from cameras, implement the visual network, and transmit high-level signals to the robot's CPG. The robot head was enlarged and circular apertures were introduced, allowing the cameras (either frame-based or event-based interchangeably) to observe the robot's surroundings. A set of acrylic domes were used as waterproof camera housing. To prevent water from leaking into the robot's head, a modular bumper system was introduced. These bumpers protected the domes from cracking in the event of collisions with the sides of the pool. An aluminum base plate was designed to carry and cool the robot's embedded PC (NanoPi). This was found to appreciably reduce the CPU's working temperature from $45^{\circ} \mathrm{C}$ to $30^{\circ} \mathrm{C}$. The finalized Envirobot design can be seen in Fig. 1a, while a demonstration of the bumper installation is shown in Fig. 1c.

\section{B. Frame-Based Object Detection}

A couple of XIMEA MU9PC-MH RGB cameras were used to validate the visuomotor control network proposed in [12]. Images were captured using a pair of cameras fitted with wideangle fish-eye lenses. The cameras were placed such that they each formed a $20^{\circ}$ angle with the sagittal (longitudinal) plane of the robot's head. Each sensor was equipped with a $180^{\circ}$ fish-eye lens, yielding a $320^{\circ}$ composite field of view. This configuration was identical for both frame-based and eventbased cameras. A color-based blob detection was used to classify objects in the robot's field of view as predators, prey, or obstacles. Each captured image was first projected to the HSV color space. For each color of interest, hue thresholds were incremented between a minimum and maximum value. The application of each threshold generated a binary mask that was applied to the image, eliminating pixels of the undesired color. Homogeneous sets of pixels were then grouped into units called blobs. Following this, all blobs corresponding to the same color were compared, and those within a specified distance were merged into single units. This image analysis technique was also how objects' sizes and positions were estimated. In this way, the output of the blob detection served as the input to the neural network (as described in sections II-E and II-F). All image processing was performed using functions provided by the OpenCV library (v3.3.1) [14].

\section{Event-Based Object Detection}

A pair of iniVation mini-eDVS cameras were used as the event-based sensors. As with the frame-based cameras, wideangle fish-eye lenses were fitted to the mini-eDVS devices, affording the system an approximately $240^{\circ}$ field of vision.

Rather than capturing full images at a fixed frame rate, this hardware allows individual pixels to fire events in response to light intensity changes. Each of the $128 \times 128$ pixels in a Dynamic Vision Sensor (DVS) was projected to one of the neurons in the network's 20x1 neuron input layer. Since color detection was not possible with these devices, a blink-detection algorithm was used to label objects in the robot's view. A simplified version of the algorithm for flash detection in [15] was adopted that omitted the use of a particle filter. Rather than estimating the location of flashing objects, any pixels measured to be flashing at $86 \mathrm{~Hz}$ were used to directly stimulate the input layer of the neural network.

\section{Experimental Setup}

In order to test the behavior and performance of the system, we constructed a water-proof target, as seen in Fig. 1b, that was fitted with a small LED on top for compatibility with the overhead LED tracking system. For frame-based cameras, the bulb's color served as the stimulus (red for predator, green for prey). However, the dynamic vision sensors provided no color information. To overcome this limitation, targets were made to flash at a constant frequency of $86 \mathrm{~Hz}$, and were detected through a blink-tracking algorithm based on [15]. Flashing pixels were used to directly stimulate the neuron network's input layer for the attractive behavior when DVS hardware was fitted. For the performance comparison, only the approach behavior was used. The overhead tracking system was able to record a minimum distance of $0.45 \mathrm{~cm}$ between the target and the Envirobot. This was due to the placements of the LEDs on the robot and target bulb as illustrated in Fig. 1c. 


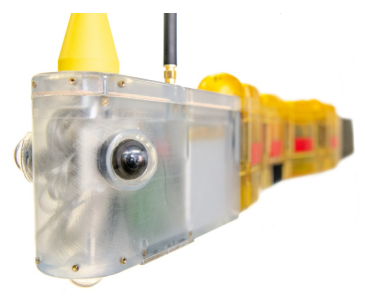

(a)

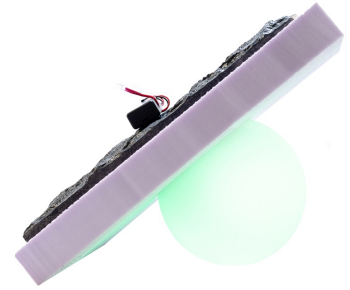

(b)

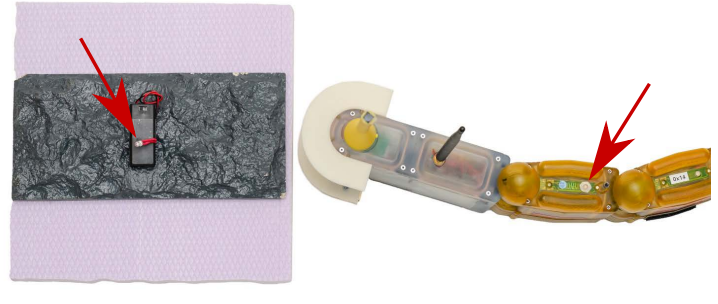

(c)

Fig. 1. The Envirobot target bulb and overhead tracking LEDs. (a) The updated vision-ready Envirobot system. Antennas are used for remote reprogramming. The new head is connected to four modules making up the robot's spine, which were designed in [13]. (b) The target bulb along with corresponding tracking LED. A Styrofoam platform and weight were used to ensure that the bulb remained completely submerged in the water. (c) Top view of the minimum distance between the platform LED (right red arrow) and the Envirobot tracking LED (left red arrow) is $0.45 \mathrm{~cm}$ during collision.

\section{E. Neuron Model}

The neuron implementation adopted for this design was based on the leaky integrate-and-fire (LIF) neuron model [16]. The LIF representation is a simplification that captures the overall functionality of biological neurons. The advantage of this simplified model is that it achieves this with significantly less parametric complexity than biophysical models such as that developed by Hodgkin and Huxley [17].

A leaky integrate-and-fire neuron can be represented in terms of the electrical properties of its membrane. An LIF neuron's membrane can be described by its resting potential $m_{o}$, its capacitance $C_{m}$, and its resistance $R_{m}$, and may be stimulated by an input current $I_{i}(t)$. These factors directly affect the membrane potential $m(t)$, which behaves according to the first order ordinary differential equation used to describe RC circuits [18].

$$
R_{m} C_{m} \frac{d m(t)}{d t}=-\left[m(t)-m_{o}\right]+R_{m} I_{i}(t)
$$

The number of parameters in this model were reduced by defining the membrane's time constant as $\tau_{m}=R_{m} C_{m}$. We also applied Ohm's law to to the input current, yielding an input membrane potential $m_{i}(t)=R_{m} I_{i}(t)$. The resting potential of all neurons in our network were set to $m_{o}=0$, and a constant refractory period of $T_{\text {refractory }}=1 \mathrm{~ms}$ was specified. A modification to the LIF model was introduced in order to prevent $m_{i}$ from remaining charged due to outdated stimuli. A forgetting factor $\alpha=0.1$ was used to drive $m_{i}$ to zero if large periods of time elapsed without stimulation.

$$
\hat{m}_{i}(t)=\operatorname{Re} L U\left(m_{i}(t)-\alpha\right)
$$

The implementation of each neuron in the network was represented by

$$
\tau_{m} \frac{d m(t)}{d t}=-\left[m(t)-m_{o}\right]+\hat{m}_{i}(t)
$$

The membrane potential was computed in real-time by continuously solving the ODE using the Euler method [19].

\section{F. Neural Network}

The visuomotor control implemented in this project was based on the work presented by Sarvestani et al. [12]. The neuron network was comprised of three main units: a stimulus prioritization block, a behavior arbitration block, and an output generation block. These subsystems allowed the platform to filter competing signals, select the optimal behavior, and drive the robot CPG accordingly. An overview of the signal flows in this system is presented in Fig. 2.

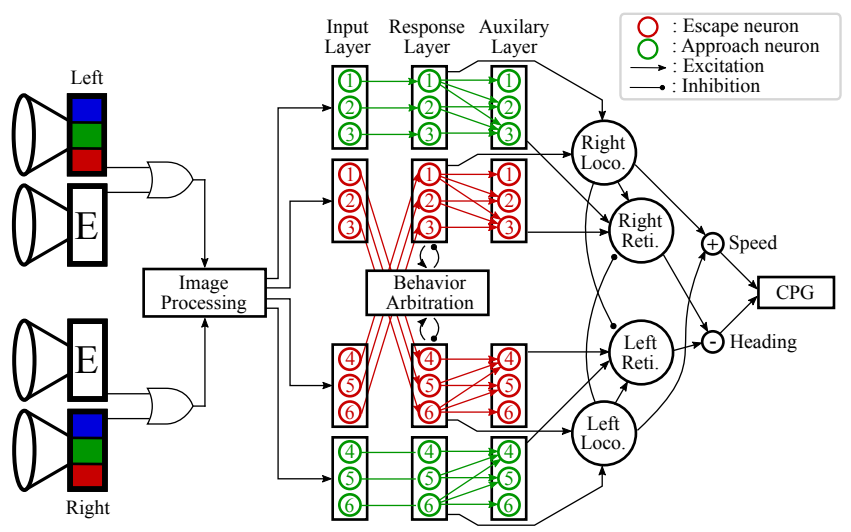

Fig. 2. A system overview depicting the relationships between the cameras, the neural sub-networks, and the central pattern generator used to drive the robot. Either frame-based or event-based cameras can be used to stimulate the neural network. This input is processed and then fed into the the neural network. The interactions between the neural network's subsystems allow the robot to prioritize between stimuli of the same type and to arbitrate between stimuli triggering different behaviors. The result of the processing done by the network is used to drive the heading and speed of a central pattern generator.

For all equations describing the network connections, $m_{i}(t)$ represents neuron input potential, $m(t)$ represents neuron membrane potential, $w$ is the connection strength between two neurons, and $N$ is the total number of neurons in each layer. $B$ is the set of all behaviors that the robot may perform. The computational complexity of the neural network is $\mathrm{O}\left(n^{2}\right)$, where $\mathrm{n}$ is the width of the input, response, and auxiliary layers of the neural network.

1) Stimulus Prioritization \& Position Encoding: The stimulus prioritization strategy was modeled on the structure of the optic tectum found in vertebrate organisms [20], and replicates their ability to select for the most salient among competing visual stimuli via inhibitory tectal circuits [21][22]. For example, when presented with numerous predators, this subsystem allowed the robot to identify which enemy pre- 
sented the most immediate risk. The sub-network also served as a transformation between stimuli positions in the robot's field of view and system turning strength.

The stimulus prioritization sub-networks each consisted of an "Input Layer", a "Response Layer", and an "Auxiliary Layer" for each possible behavior. The neurons of this subsystem were organized into one of two possible configurations; the first arrangement drove the robot towards attractive stimuli, while the other layout was for repulsive stimuli.

For all types of behavioral circuits, the instantaneous lateral inhibition between response layer neurons is defined as $I(t)$ in

$$
\begin{aligned}
& I_{b, n}(t)=w^{\text {Resp-Resp }} \sum_{k=1, k \neq n}^{N} m_{b, k}^{\text {Resp }}(t), \\
& \forall n \epsilon[1, N], \quad \forall b \in B .
\end{aligned}
$$

For visual clarity, these lateral inhibitions are not depicted in Fig. 2. The connections within behavioral circuits used to direct the robot towards attractive stimuli (prey) obeyed the following rules.

$$
\begin{gathered}
m_{i_{b, n}}^{\text {Resp }}(t)=w_{b, n}^{\text {In-Resp }} m_{b, n}^{\text {In }}(t)-I_{b, n}(t), \\
\forall n \epsilon[1, N], \quad \forall b \epsilon B, \\
m_{i_{b, n}}^{\text {Aux }}(t)=\left\{\begin{array}{c}
\sum_{k=1}^{n} w_{b, k}^{\text {Resp }-A u x} m_{b, k}^{\text {Resp }}(t) \\
n \epsilon[1, N / 2], \quad \forall b \epsilon B, \\
\sum_{k=n}^{N} w_{b, k}^{\text {Resp }-A u x} m_{b, k}^{\text {Resp }}(t) \\
n \epsilon(N / 2, N], \quad \forall b \epsilon B .
\end{array}\right.
\end{gathered}
$$

The connections within behavioral circuits used to direct the robot away from repulsive stimuli (predators and obstacles) were defined in

$$
m_{i_{b, n}}^{\text {Resp }}(t)=\left\{\begin{array}{r}
w_{b, n}^{I n-R e s p} m_{b, n-\frac{N}{2}}^{I n}(t)-I_{b, n}(t) \\
n \epsilon(N / 2, N], \quad \forall b \epsilon B \\
w_{b, n}^{I n-R e s p} m_{b, \frac{N}{2}+n}^{I n}(t)-I_{b, n}(t) \\
n \epsilon[1, N / 2], \quad \forall b \in B
\end{array}\right.
$$

The connections between neurons of the Response and Auxiliary layers are identical to Eq. (6).

All neuron connections in this sub-network were made according to equations Eq. (5)-Eq. (7), and are exemplified in Fig. 2 for a 6-neuron-wide network (that is, each behavior is implemented by 6 neurons).

2) Behavior Arbitration: The behaviour arbitration subnetwork allows the controller to designate a single response when faced with competing behavioral stimuli. The simplified model is comprised of three distinct neurons for each behavioral circuit. This processing block allows the system to attenuate the contribution of less pertinent behavioral cues to the system output. Drawing from the anatomy of the basal ganglia (the neural structure on which this sub-network is based), these three neurons were labeled as the subthalamic nucleus (STN) neuron, internal globus pallidus (GPi) neuron, and external globus pallidus (GPe) neuron [23].

The STN neurons receive stimulation from the response neurons and are inhibited by the GPi neuron of the same behavioral circuit. The STN neurons are also inhibited by GPe neurons of all other behaviors. It can be formulated as

$$
\begin{aligned}
m_{i_{b}}^{S T N}(t)= & +\sum_{n=1}^{N} w_{b, n}^{R e s p-S T N} m_{b, n}^{R e s p}(t) \\
& -w_{b}^{G P i-S T N} m_{b}^{G P i}(t) \\
& -\sum_{k \in B, k \neq b} w_{k}^{G P e-S T N} m_{k}^{G P e}(t), \quad \forall b \in B .
\end{aligned}
$$

Each GPe neuron is excited by the STN neurons of the same behavioral circuit.

$$
m_{i_{b}}^{G P e}(t)=w_{b}^{S T N-G P e} m_{b}^{S T N}(t), \quad \forall b \epsilon B
$$

The GPi neuron of a given behavior is stimulated by the STN neurons of all other behaviors. Each GPi neuron is also inhibited by the GPe neuron of the same behavior.

$$
\begin{aligned}
m_{i_{b}}^{G P i}(t)= & +\sum_{k \in B, k \neq b} w_{k}^{S T N-G P i} m_{k}^{S T N}(t) \\
& -w_{b}^{G P e-G P i} m_{b}^{G P e}(t), \quad \forall b \epsilon B .
\end{aligned}
$$

Finally, the GPi neuron of each behavior inhibits all response layer neurons of the same behavior.

$$
m_{i_{b, n}}^{\text {Resp }}(t)=-w_{b, n}^{G P i-\operatorname{Resp}} m_{b}^{G P i}(t), \quad \forall n \epsilon[1, N], \quad \forall b \epsilon B .
$$

The stimulative and inhibitory connections between the neurons of this sub-network were formally represented in equations Eq. (8)-Eq. (11). The circuit is illustrated for a system capable of performing 3 different behaviors in Fig. 3 . Although only 2 behaviors were tested in this work (approach and escape), the 3 behavioral circuits described in [12] are depicted in Fig. 3 to show how the architecture generalizes to support multiple behaviors.

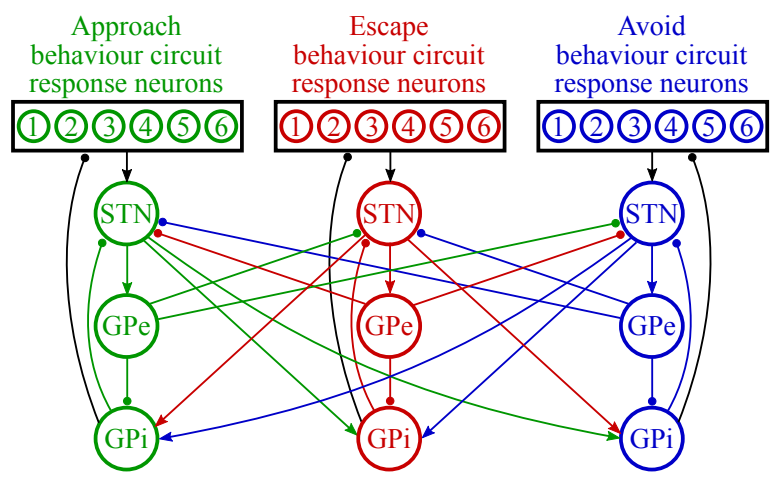

Fig. 3. The architecture of the behavior arbitration sub-network. Each behavior has an associated STN, GPe, and GPi neuron. The module receives input stimulation from the response layer neurons. The output of this artificial basal ganglia are the inhibitory connections between each GPi neuron and all response layer neurons of the same behavior. The connections of a third behavior (avoidance) are drawn here in order to show how the arbitration network can generalize beyond two behaviors. 
3) Network Output: The final portion of the network generates the robot's CPG drive signals. In this unit of the network, a slight modification to the architecture of [12] is proposed; the swimming speed is controlled by the locomotor neurons' combined firing rates, while turning is dictated by the difference in reticulospinal neuron activations. Additionally, the output of each locomotor neuron is made to inhibit the reticulospinal neuron of the opposite side, further emphasizing this difference. This sub-unit has been implemented as described by

$$
\begin{aligned}
& m_{i_{l e f t}}^{\text {Loco }}(t)=\sum_{b \in B} \sum_{n=1}^{\frac{N}{2}} w_{b, n}^{\text {Resp-Loco }} m_{b, n}^{\text {Resp }}(t) . \\
& m_{i_{\text {right }}}^{\text {Loco }}(t)=\sum_{b \in B} \sum_{n=\frac{N}{2}+1}^{N} w_{b, n}^{\text {Resp-Loco }} m_{b, n}^{\text {Resp }}(t) . \\
& m_{i_{\text {left }} \text { Reticulo }}(t)=+\sum_{b \in B} \sum_{n=1}^{\frac{N}{2}} w_{b, n}^{A u x-\text { Reticulo }} m_{b, n}^{A u x}(t) \\
& +m_{i_{\text {left }}}^{\text {Loco }}+m_{i_{\text {right }}}^{\text {Loco }} . \\
& m_{i_{\text {right }}}^{\text {Reticulo }}(t)=+\sum_{b \in B} \sum_{n=\frac{N}{2}+1}^{N} w_{b, n}^{\text {Aux-Reticulo }} m_{b, n}^{\text {Aux }}(t) \\
& +m_{i_{\text {left }}}^{\text {Loco }}+m_{i_{\text {right }}}^{\text {Loco }} .
\end{aligned}
$$

Fig. 2 illustrates these connections. The instantaneous estimates of speed and heading were determined using

$$
\begin{gathered}
\text { speed }(t)=R\left(x_{\text {right }}^{\text {Loco }}(t)\right)+R\left(x_{\text {left }}^{\text {Loco }}(t)\right) \\
\text { heading }(t)=R\left(m_{i_{\text {right }}^{\text {Reticulo }}(t)}^{\text {Reticulo }}(t)\right)
\end{gathered}
$$

The function $R$ is the rectifier (activation function) used to map neuron outputs to values capable of driving the CPG.

\section{RESULTS}

\section{A. Behavioral Tests}

To experimentally validate the visuomotor control architecture proposed in [12], the controller was used to guide the Envirobot through seven different scenarios. For these experiments, only frame-based cameras were used and behaviors were produced in response to color cues. Using the color-based blob detection algorithm described in II-B, green circles were interpreted as prey and red circles were considered predators. The robot was made to operate at a slow and fixed frequency in order to avoid damaging the system.

The approach behavior was tested by placing the Envirobot in a pool with a solitary attractive stimulus (Fig. 4a). Likewise, the escape behavior was evaluated by recording the robot's reaction to a single repulsive stimulus (Fig. 4b). We then studied the system's ability to prioritize between different stimuli of the same behavioral group. This was done by exposing the platform to two attractive stimuli simultaneously (Fig. 4c). Finally, the behavior arbitration was studied by placing attractive and repulsive stimuli in close proximity (Fig. 4d). The results of these four experiments are presented in Fig. 4.
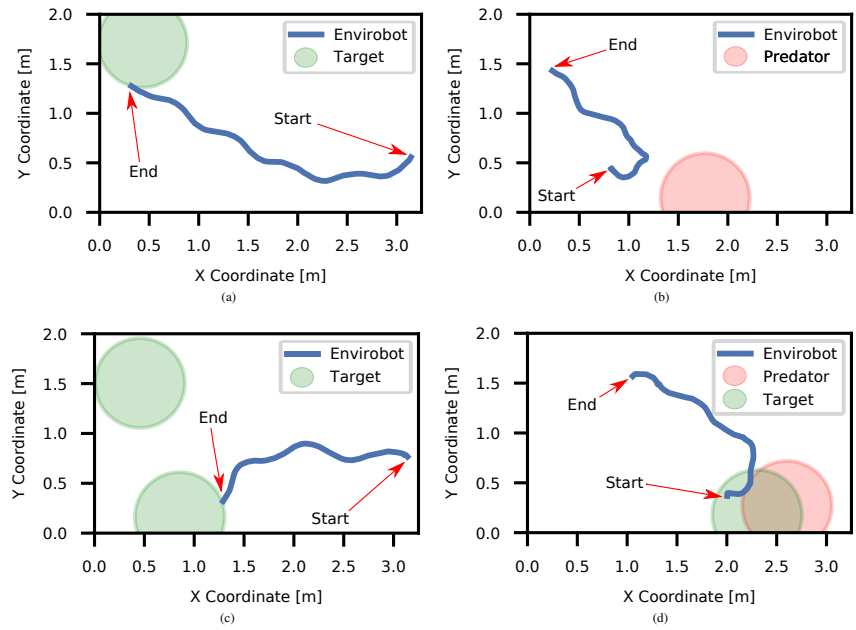

Fig. 4. The experimental Envirobot swimming trajectories in four distinct scenarios. These experiments were conducted by placing the robot in a pool with one or more stimuli, and then tracking its trajectory during locomotion. The robot was presented with (a) a single attractive stimulus (prey), (b) a single repulsive stimulus (predator), (c) two attractive stimuli (prey), but at different locations to test the controller's stimulus prioritization capabilities. Finally, (d) the robot was presented with an attractive stimulus (prey) and a neighboring repulsive stimulus (predator) to test the controller's behavior arbitration capabilities.

The robot was then made to navigate through three dynamic scenarios. Exposing the system to solitary attractive and repulsive stimuli in constant motion verified the network's ability to drive chasing and fleeing behaviors respectively. In a more complex scenario, we studied the platform's response to sudden changes in its environment. The scenario began by initially presenting the robot with two attractive stimuli at different distances, similar to the case in Fig. 4c. However, upon approaching its initial target, a predator was suddenly made to appear. The Envirobot was capable of changing its behavior to first escape the predator, and then target the safer prey. The chasing behavior, fleeing behavior, and trajectory readjustment behavior were recorded and made available for viewing as a supplementary video to this work.

\section{B. DVS Performance}

We characterized the performance of the robot when driven by dynamic vision sensors instead of frame-based cameras. The number of complete passes through the neuron network per second were measured using both cameras to provide an objective measure of the computational advantage introduced by the bio-inspired vision strategy. A higher number of passesper-second is desirable, as this corresponds to a network being able to process visual input data more quickly. The controller was run while the robot was exposed to no stimuli, one stimulus, and two stimuli. Each scenario was maintained for 60 seconds, and repeated for both cameras. The results presented in Fig. 5 indicate a dramatic improvement offered by the event-based strategy. We observed an order-of-magnitude improvement when using the dynamic vision sensors.

A final experiment studied the improvement in robot swimming precision due to the dynamic vision sensors. 20 experiments (10 with frame-based cameras, and 10 with event- 


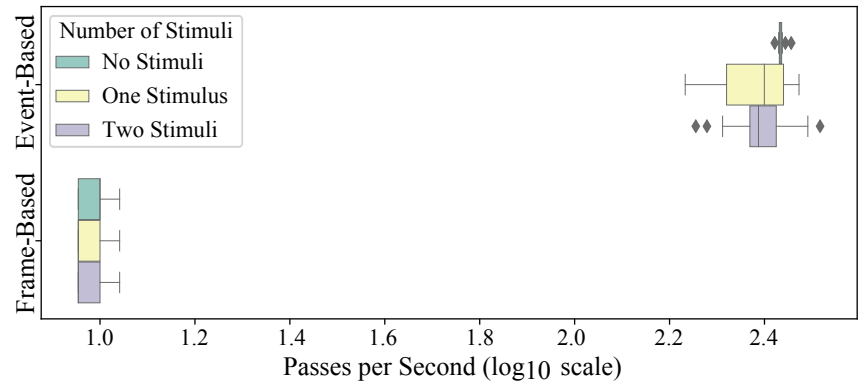

Fig. 5. A comparison between the neuron network computation rates achieved by the two types of image capture. The controller was run for a duration of three minutes for each type of camera. Tests began with no visual stimuli, and a stimulus was added to the robot's field of view each minute. The mean computation rates for the event-based cameras were measured to an order of magnitude higher than their frame-base counterparts for each scenario.

based cameras) were performed in which the robot was made to attack a prey from a starting point approximately 3 meters away. Experiments were allowed to run until either the platform reached its target or collided with one of the pool's walls.

A metric was developed to evaluate robot's target approach performance. At every time instant, the angle formed by the robot's current location, the target location (the angle vertex), and its most recent past location was computed. This gaze alignment angle, $\theta_{\text {gaze }}(t)$, represented how wellcentered within the robot's field of view the target was at each time instant. Angles closer to $0^{\circ}$ represented strong instantaneous alignment, while angles greater in magnitude indicated a deviation from head-on approach. Each angle was multiplied by a normalizing factor, defined as the ratio between the current distance-to-target and the initial distance-to-target. To quantify the robot's performance in a single trial, the gaze angles were integrated over time. The resulting value was coined the cumulative gaze misalignment, $M_{\text {gaze }}\left[{ }^{\circ} \mathrm{s}\right]$. This quantity represented the amount of time spent with the target not centered within the robot's field of view. In other words, this served as a measure of how long the robot was able to keep the object dead-ahead.

Fig. 6 illustrates the trajectories for the two camera types, while Fig. 7a depicts the evolution of the gaze alignment for each trajectory. Statistics for the 20 experiments are reported in Fig. 7b. The results clearly favored event-based cameras for the prey approach tasks. Use of dynamic vision sensors led to a reduction in the cumulative gaze misalignment, falling from an average of $\bar{M}_{g a z e}=3.34^{\circ} \mathrm{s}$ to $\bar{M}_{g a z e}=0.57^{\circ} \mathrm{s}$.

\section{DISCUSSION}

\section{A. Visual Memory}

The visuomotor controller presented in this work was only capable of generating output signals based on instantaneous input. While the use of event-based cameras improved the robot's head-on approach of attractive stimuli, the purely reactive nature of the controller made it impossible to respond to stimuli outside of its field of view. For example, the robot was found to cease performing its escape behavior upon successfully turning away from a predator such that visual

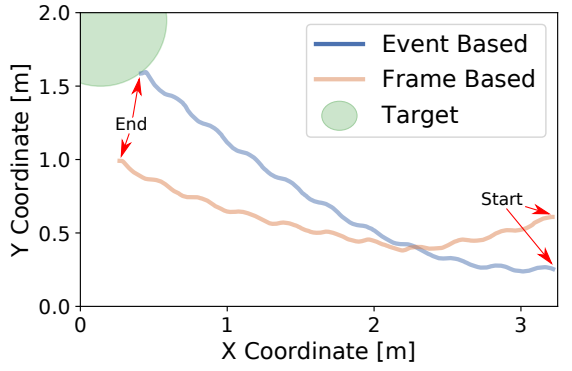

Fig. 6. An example of approach trajectories for both vision systems recorded during the experiments. The robot was placed 3 meters away from the target and allowed to perform its approach behavior. Experiments were run until the robot reached the target, or until collision with the bounds of the pool. In the trial shown here, the frame-based trajectory was unable to produce sufficient turning to reach the target before colliding with the pool's perimeter.

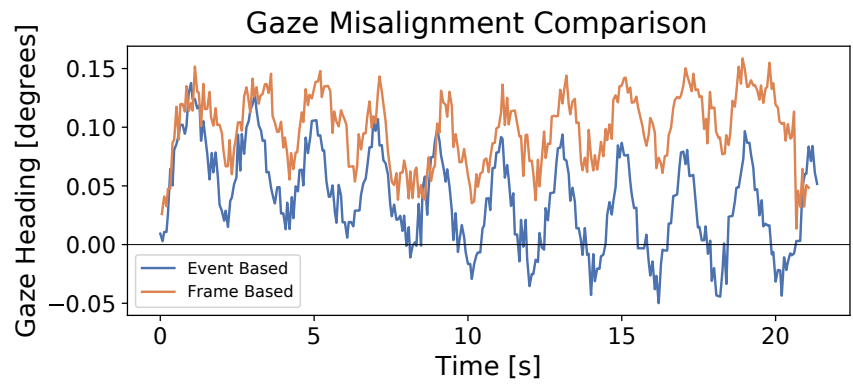

(a)

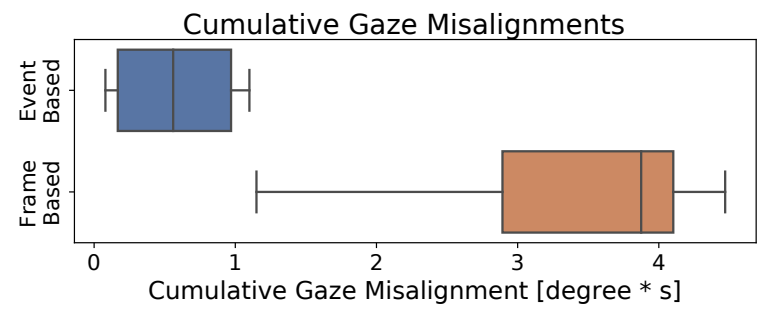

(b)

Fig. 7. A performance comparison between event-based and frame-based cameras. (a) The gaze misalignment plots corresponding to the two trajectories of Fig. 6. The area under each curve was used as a final measure of how well the robot performed. An area of $4.5^{\circ} \mathrm{s}$ was recorded for the frame-based trial. The event-based camera experiment exhibited a significant improvement, with an cumulative gaze misalignment of $0.8^{\circ} \mathrm{s}$. (b) The cumulative gaze misalignment scores for the two types of camera shows a significant improvement in the robot's ability to align the target with its heading when the event-based cameras were used. The mean of $\bar{M}_{\text {gaze }}=3.34^{\circ} \mathrm{s}$, obtained using framebased cameras, dropped to $\bar{M}_{\text {gaze }}=0.57^{\circ} \mathrm{s}$ with event-based cameras.

contact was broken. Additionally, the controller lacked an internal model of the robot's anguilliform swimming gait. Due to stimuli rapidly shifting between the right and left eye, the platform sometimes overshot its objective.

In vertebrates, the hippocampus helps to solve this memory problem by maintaining a spatial map of its organism's surroundings [24]. Biologically inspired vision systems seek to reproduce the functionality of such spatial maps [25] and visual short-term memory [26]. Kinaesthesia, on the other hand, allows organisms to estimate the relative locations and orientations of body parts. It may be possible to improve our controller by extending the visuomotor network to include a more advanced localization and memory system. This could 
enhance the robot's precision while attacking prey, and allow it to retain an awareness of stimuli that have fallen outside of its field of view.

\section{B. Parameter Optimization}

Another limitation of this platform is the lack of a formal method for optimizing the parameters of the neural network. The framework was tuned heuristically, which was made possible by enforcing several model simplifications; all synaptic weights were fixed to a value of $w=1$, all forgetting factors to $\alpha=0.1$, and all refractory periods to $T_{\text {refractory }}=1 \mathrm{~ms}$. It may be possible to improve the robot's performance in terms of speed and precision by using a more rigorous parameter optimization strategy.

\section{Behavior Adaptation \& Learning}

It may be interesting to study the ability of this controller to generalize its performance to previously unknown stimuli. Lamprey and other organisms have been found to be capable of adapting their behaviors based on danger cues sensed in their surrounding environments [27]. Damage-released alarm cues originating from conspecific organisms under distress mark regions as high-risk, and can instigate the animals' alarm responses [28]. Experiments have even shown that certain fish species are able to learn from such odor cues, which are believed to play a role in teaching organisms to recognize new entities as predators. It has been found that combining damage released alarm cues with visual information produces learned predator recognition [29].

Using such cues as penalties or rewards, it should be possible to generalize the controller's performance when presented with novel objects. In aquatic environments, chemical signals such as the aforementioned danger cues, are dispersed by Brownian motion over time. Such signals may not even be visually detectable, making them poor candidates for visual tracking (or avoidance). However, they could prove valuable as a means for providing positive reinforcement to help form new network connections. This could be done in an approach resembling the policy optimization algorithms commonly used in reinforcement learning.

Additionally, recent research has found that locusts are capable of exploiting directional visual cues to rapidly adapt their escape behaviors [30]. This has been achieved using purely environmental information, without exposure to any tangible rewards or penalties. Similarly to chemical danger signals, contextual visual data could also be used to extend the controller's performance. The visual recognition of social information (for example, an escaping school of fish) may be used to train new controller networks in order to associate a novel entity with a behavioral response.

Such learning algorithms could have interesting applications for environmental monitoring systems. Deploying robots capable of sensing and intelligently interpreting contextual cues could prove to be an asset in discovering and localizing newly introduced pollutants.

\section{Underwater use of Event-Based Cameras}

This first use of event-based cameras in an underwater environment was shown to have a positive effect on the precision of goal directed swimming in the Envirobot platform. However, to simplify the challenge of underwater object detection, these experiments were conducted with a single attractive stimulus and used a flashing target. Indeed, aquatic robot vision remains a challenge due to the effect of the medium on the dispersion and attenuation of light. These effects manifest in a significantly reduced depth of field, limiting the utility of cameras in underwater monitoring and exploration applications. Additional work is required to rigorously characterize the performance of event-based cameras in water, and to develop algorithms capable of directing robot locomotion in more complex environments.

\section{CONClusion}

The objectives of this work were to validate a biologically inspired visuomotor controller, and serve as the first use case of event driven cameras underwater. To achieve this, a visionready head was designed for use with a modular lamprey robot. The control architecture was then evaluated on its ability to reproduce the behaviors predicted in [12]. Finally, the performance benefits associated with the use of event-based, rather than frame-based, cameras were recorded.

The model was successful in reproducing approach behavior (attacking prey) and escape behavior (fleeing predators), while prioritizing between various stimuli of the same behavioral groups. The system was also found effective in arbitrating between competing behaviors. Based on the structure of vertebrate neurovisual circuits, this controller was capable of producing complex behaviors in response to visual cues.

Following this, event-based sensors were used to drive simple goal directed swimming, and system performance was compared with that obtained using frame-based cameras. It was found that the biologically inspired vision hardware improved the Envirobot's ability to approach prey with reduced overshoot. This instrument also enabled the CPU to process the neuron network more rapidly, allowing the leaky integrateand-fire neurons to adopt more biologically accurate parameter values. The challenges associated with this hardware are an inability to detect color, a lack of algorithms for image rendering in aquatic environments, and a limited understanding of the device performance underwater.

This work offers contributions to the field of robotics while simultaneously presenting a tool for future studies in neurobiology. The vision-ready Envirobot offers an ideal platform to investigate vision-controlled locomotion in anguilliform swimmers such as salamanders and lamprey. By their very nature, the dynamic vision sensors used in this study are ideal for researching how optic-flows are used by organisms to direct swimming. At the same time, this work contributes to the state-of-the art in underwater robotics. The computational and sensory advantages offered by the DVS devices may lead to improvements in applications such as marker tracking on active beacons, environment monitoring, or search and rescue systems. 


\section{REFERENCES}

[1] Z. Gao, Q. Shi, T. Fukuda, C. Li, and Q. Huang, "An overview of biomimetic robots with animal behaviors," Neurocomputing, vol. 332, pp. $339-350,2019$.

[2] D. Floreano, A. J. Ijspeert, and S. Schaal, "Robotics and neuroscience," Current Biology, vol. 24, no. 18, pp. R910 - R920, 2014.

[3] A. J. Ijspeert, "Central pattern generators for locomotion control in animals and robots: A review," Neural Networks, vol. 21, no. 4, pp. $642-653,2008$, robotics and Neuroscience.

[4] J. Sattar, P. Giguere, G. Dudek, and C. Prahacs, "A visual servoing system for an aquatic swimming robot," in 2005 IEEE/RSJ International Conference on Intelligent Robots and Systems, Aug 2005, pp. 14831488.

[5] Y. Hu, W. Zhao, L. Wang, and Y. Jia, "Underwater target following with a vision-based autonomous robotic fish," in 2009 American Control Conference, June 2009, pp. 5265-5270.

[6] L. Manfredi, T. Assaf, S. Mintchev, S. Marrazza, L. Capantini, S. Orofino, L. Ascari, S. Grillner, P. Wallén, Ö. Ekeberg, C. Stefanini, and P. Dario, "A bioinspired autonomous swimming robot as a tool for studying goal-directed locomotion," Biological Cybernetics, vol. 107, no. 5, pp. 513-527, Oct 2013.

[7] J. Yu, F. Sun, D. Xu, and M. Tan, "Embedded vision-guided 3-d tracking control for robotic fish," IEEE Transactions on Industrial Electronics, vol. 63, no. 1, pp. 355-363, Jan 2016.

[8] A. J. Ijspeert, A. Crespi, D. Ryczko, and J.-M. Cabelguen, "From swimming to walking with a salamander robot driven by a spinal cord model," Science, vol. 315, no. 5817, pp. 1416-1420, 2007.

[9] J. Yu, M. Tan, J. Chen, and J. Zhang, "A survey on cpg-inspired control models and system implementation," IEEE Transactions on Neural Networks and Learning Systems, vol. 25, no. 3, pp. 441-456, March 2014.

[10] P. Lichtsteiner, C. Posch, and T. Delbruck, "A $128 \times 128120 \mathrm{db} 15 \mu \mathrm{s}$ latency asynchronous temporal contrast vision sensor," IEEE Journal of Solid-State Circuits, vol. 43, no. 2, pp. 566-576, Feb 2008.

[11] E. Mueggler, N. Baumli, F. Fontana, and D. Scaramuzza, "Towards evasive maneuvers with quadrotors using dynamic vision sensors," in 2015 European Conference on Mobile Robots (ECMR), Sep. 2015, pp. $1-8$.

[12] I. Kamali Sarvestani, A. Kozlov, N. Harischandra, S. Grillner, and Ö. Ekeberg, "A computational model of visually guided locomotion in lamprey," Biological Cybernetics, vol. 107, no. 5, pp. 497-512, Oct 2013.

[13] B. Bayat, A. Crespi, and A. Ijspeert, "Envirobot: A bio-inspired environmental monitoring platform," in 2016 IEEE/OES Autonomous Underwater Vehicles (AUV), Nov 2016, pp. 381-386.

[14] G. Bradski, "The OpenCV Library," Dr. Dobb's Journal of Software Tools, 2000

[15] A. Censi, J. Strubel, C. Brandli, T. Delbruck, and D. Scaramuzza, "Lowlatency localization by active led markers tracking using a dynamic vision sensor," in IEEE/RSJ International Conference on Intelligent
Robots and Systems (IROS), Tokyo,Japan, November 2013, pp. 891898.

[16] L. Lapicque, "Recherches quantitatives sur l'excitation electrique des nerfs traitée comme une polarization," J. Physiol. Pathol., vol. 9, pp. 620-635, 1907.

[17] F. Santamaria and J. Bower, "Hodgkin-huxley models," in Encyclopedia of Neuroscience, L. R. Squire, Ed. Oxford: Academic Press, 2009, pp. $1173-1180$.

[18] W. Gerstner, W. Kistler, R. Naud, and L. Paninski, Neuronal Dynamics: From Single Neurons to Networks and Models of Cognition. Cambridge University Press, 2014.

[19] D. F. Griffiths and D. J. Higham, Euler's Method. London: Springer London, 2010, pp. 19-31.

[20] I. H. Bianco and F. Engert, "Visuomotor transformations underlying hunting behavior in zebrafish," Current Biology, vol. 25, no. 7, pp. 831846, Mar 2015.

[21] A. A. Kardamakis, K. Saitoh, and S. Grillner, "Tectal microcircuit generating visual selection commands on gaze-controlling neurons," Proceedings of the National Academy of Sciences, vol. 112, no. 15, pp. E1956-E1965, 2015.

[22] S. P. Mysore, A. Asadollahi, and E. I. Knudsen, "Global inhibition and stimulus competition in the owl optic tectum," Journal of Neuroscience, vol. 30, no. 5, pp. 1727-1738, 2010.

[23] E. R. Kandel, J. H. Schwartz, T. M. Jessell, S. A. Siegelbaum, and A. J. Hudspeth, Principles of Neural Science, Fifth Edition. McGraw-Hill, 2013.

[24] J. O'keefe and L. Nadel, The hippocampus as a cognitive map. Oxford: Clarendon Press, 1978.

[25] K. Rebai, O. Azouaoui, and N. Achour, "Bio-inspired visual memory for robot cognitive map building and scene recognition," in 2012 IEEE/RSJ International Conference on Intelligent Robots and Systems, Oct 2012, pp. 2985-2990.

[26] J. Vega, E. Perdices, and J. M. Cañas, "Robot evolutionary localization based on attentive visual short-term memory," Sensors (Basel), vol. 13, no. 1, pp. 1268-1299, Jan 2013.

[27] C. M. Wagner and J. D. Bals, "Behavioral responses of sea lamprey (petromyzon marinus) to a putative alarm cue derived from conspecific and heterospecific sources," Behaviour, vol. 149, no. 9, pp. 901 - 923, 2012.

[28] J. B. Hume and M. Wagner, "A death in the family: Sea lamprey (petromyzon marinus) avoidance of confamilial alarm cues diminishes with phylogenetic distance," Ecology and Evolution, vol. 8, no. 7, pp. 3751-3762, 2018

[29] G. E. Brown, M. C. O. Ferrari, and D. P. Chivers, Learning about Danger: Chemical Alarm Cues and Threat-Sensitive Assessment of Predation Risk by Fishes. John Wiley \& Sons, Ltd, 2011, ch. 4, pp. 59-80.

[30] D. Romano, G. Benelli, and C. Stefanini, "Encoding lateralization of jump kinematics and eye use in a locust via bio-robotic artifacts," Journal of Experimental Biology, vol. 222, no. 2, 2019. 